\title{
Variational Statement and Domain Decomposition Algorithms for Bitsadze-Samarskii Nonlocal Boundary Value Problem for Poisson's Two-Dimensional Equation
}

\author{
Temur Jangveladze, ${ }^{1,2}$ Zurab Kiguradze, ${ }^{1}$ and George Lobjanidze ${ }^{3}$ \\ ${ }^{1}$ Ilia Vekua Institute of Applied Mathematics of Ivane Javakhishvili, Tbilisi State University, 2 University Street, 0186 Tbilisi, Georgia \\ ${ }^{2}$ Georgian Technical University, 77 Kostava Avenue, 0175 Tbilisi, Georgia \\ ${ }^{3}$ Caucasus University, 10 Politkovskaya Street, 0186 Tbilisi, Georgia \\ Correspondence should be addressed to Temur Jangveladze; tjangv@yahoo.com
}

Received 10 February 2014; Accepted 4 June 2014; Published 19 June 2014

Academic Editor: Athanasios N. Yannacopoulos

Copyright (C) 2014 Temur Jangveladze et al. This is an open access article distributed under the Creative Commons Attribution License, which permits unrestricted use, distribution, and reproduction in any medium, provided the original work is properly cited.

The Bitsadze-Samarskii nonlocal boundary value problem is considered. Variational formulation is done. The domain decomposition and Schwarz-type iterative methods are used. The parallel algorithm as well as sequential ones is investigated.

\section{Introduction}

In applied sciences different problems with nonlocal boundary conditions arise very often. In some nonlocal problems, unlike classical boundary value problems, instead of boundary conditions, the dependence between the value of an unknown function on the boundary and some of its values inside of the domain is given.

Modern investigation of nonlocal elliptic boundary value problems originates from Bitsadze and Samarskii work [1], in which by means of the method of integral equations the theorems are proved on the existence and uniqueness of a solution for the second order multidimensional elliptic equations in rectangular domains. Some classes of problems for which the proposed method works are given.

Many works are devoted to the investigation of nonlocal problems for elliptic equations (see, e.g., [2-18] and references therein).

It is known how a great role takes place in the variational formulation of classical and nonlocal boundary value problems in modern mathematics (see, e.g., [13-15, 19-27]).

It is also well known that in order to find the approximate solutions, it is important to construct useful economical algorithms. For constructing such algorithms, the method of domain decomposition has a great importance (see, e.g., $[23,28,29])$.

In the work [6] the iterative method of proving the existence of a solution of Bitsadze-Samarskii problem for Laplace equation was proposed. This iterative method is based on the idea of Schwarz alternating method [30, pages 249-254]. It should be noted that the usage of Schwarz alternating method not only gives us the existence of a solution, but also allows finding effective algorithms for numerical resolution of such problems. By this approach the nonlocal problem reduces to classical Dirichlet problems on whole domain that yields the possibility to apply the already developed effective methods for numerical resolution of these problems. In $[7,11-13,15]$ using Schwarz alternating method and domain decomposition algorithms BitsadzeSamarskii nonlocal problem is studied for Laplace equation. The domain decomposition algorithms are more economical than the method which was proposed in [6].

In the work [6] the reduction of nonlocal problem to the sequence of Dirichlet problems is studied. For investigating author used Schwarz lemma but not domain decomposition. At first domain decomposition for Bitsadze-Samarskii nonlocal boundary value problem was introduced in [7]. In the abstract [11] the convergence of the domain decomposition 
method for the second order nonlinear elliptic equation is given. In [12] the domain decomposition sequential and parallel algorithms are fixed. In [13] the sequential and parallel iterative algorithms are given. In [13] attention is devoted to the operator decomposition method and to the possibility of the variational formulation of the problem as well. In [15] the convergence of the domain decomposition parallel algorithm is fixed. Note that in the works [7, 11-13, 15] results are mainly given without proof.

In the works $[9,10,16,21,22,31-33]$ different methods are displayed for study of nonlocal problems in the theory of ordinary differential equations and in the theory of equations with partial derivatives.

The present work is devoted to the variational formulation and domain decomposition and Schwarz-type iterative methods for Bitsadze-Samarskii nonlocal boundary value problem for Poisson's two-dimensional equation. Here we investigate the parallel algorithm as well as sequential ones. The rate of convergence is presented too.

The outline of this paper is as follows. In Section 2 for the Poisson equation in a rectangle we state Bitsadze-Samarskii nonlocal problem. In Section 3 the variational formulation of this problem is discussed. The convergence of the Schwarztype iterative sequential algorithm is studied in Section 4. The same question for parallel algorithm is considered in Section 5. In Section 6 some conclusions are given.

\section{Formulation of Problem}

In the plane $O x y$, let us consider the rectangle $G=\{(x, y) \mid$ $-a<x<0,0<y<b\}$, where $a$ and $b$ are the given positive constants. By $\partial G$ we denote the boundary of the rectangle $G$ and by $\Gamma_{t}$ the intersection of the line $x=t$ with the set $\bar{G}=$ $G \cup \partial G$.

Consider the nonlocal Bitsadze-Samarskii boundary value problem [1]

$$
\begin{gathered}
-\Delta u(x, y)=f(x, y), \quad(x, y) \in G, \\
\left.u(x, y)\right|_{\Gamma}=0, \\
\left.u(x, y)\right|_{\Gamma_{-\xi}}=\left.u(x, y)\right|_{\Gamma_{0}},
\end{gathered}
$$

where $\Delta=\partial^{2} / \partial x^{2}+\partial^{2} / \partial y^{2}$ is the Laplace operator, $\Gamma=\partial G \backslash \Gamma_{0}$, $\xi \in(0, a) ; f(x, y)$ is a given function, $f(x, y) \in C(\bar{G})$; and $u(x, y) \in C(\bar{G}) \cap C^{(2)}(G)$ is an unknown function.

Uniqueness of the solution of problem (1)-(3) follows from the extremum principle. It is known [1] that if $f$ is a continuous function on $\bar{G}$, then there exists unique regular solution of problem (1)-(3) $u(x, y) \in C^{2}(G) \cap C(\bar{G})$.

\section{Variational Statement of Problem}

We use usual $L_{2}$ and Sobolev spaces $H^{k}$ and $\stackrel{\circ}{H}^{k}$. Let us denote by $D(\bar{G})$ the vector space of all real functions satisfying the following conditions: $v(x, y)$ is defined almost everywhere on $\bar{G}$, the boundary value $v(0, y)$ is defined almost everywhere on $\Gamma_{0}$, and $v(x, y) \in L_{2}(G), v(0, y) \in L_{2}(0, b)$.
Functions $v_{1}(x, y)$ and $v_{2}(x, y)$ are assumed as the same element of $D(\bar{G})$ if $v_{1}(x, y)=v_{2}(x, y)$ almost everywhere on $\bar{G}$ and $v_{1}(0, y)=v_{2}(0, y)$ almost everywhere on $\Gamma_{0}$.

Let $\bar{Q}=\{(x, y) \mid 0 \leq x \leq \xi, 0 \leq y \leq b\}$ and $\mathscr{T}$ the operator which extends elements of $D(\bar{G})$ as follows:

$$
\mathscr{T} v(x, y)= \begin{cases}v(x, y), & \text { if }(x, y) \in \bar{G} \\ -v(-x, y)+2 v(0, y), & \text { if }(x, y) \in \bar{Q} .\end{cases}
$$

Operator $\mathscr{T}$ associates to every function $v(x, y)$ of the vector space $D(\bar{G})$ the following function $\widetilde{v}(x, y)=\mathscr{T} v(x, y)$ in such a way that the function $\widetilde{v}(x, y)-v(0, y)$ is the odd function with respect to the variable $x$ almost everywhere for almost all $y \in[0, b]$.

Let us define on vector space $D(\bar{G})$ the scalar product

$$
[v, w]=\int_{0}^{b} \int_{-\xi}^{\xi} \int_{-a}^{x} \widetilde{v}(s, y) \widetilde{w}(s, y) d s d x d y .
$$

Introducing the scalar product (5), let us denote vector space $D(\bar{G})$ by $H(\bar{G})$ where the norm is defined as follows:

$$
\|v\|_{H}^{2}=\int_{0}^{b} \int_{-\xi}^{\xi} \int_{-a}^{x} \tilde{v}^{2}(s, y) d s d x d y .
$$

The following statements take place [24].

Theorem 1. The norm defined in $H(\bar{G})$ by the formula

$$
\|v\|^{2}=\|v(x, y)\|_{L_{2(G)}}^{2}+\|v(0, y)\|_{L_{2}(0, b)}^{2}
$$

is equivalent to the norm $\|\cdot\|_{H}$.

Theorem 2. Space $H(\bar{G})$ is complete with the metric $\rho(v, w)=$ $\|v-w\|_{H}$.

Let the domain of definition of the operator $A=-\Delta$ be the vector space $D_{A}(\bar{G}) \subset H(\bar{G})$ of the elements $v(x, y)$ defined on $\bar{G}$ for which the following conditions are fulfilled:

(1) $v(x, y) \in C^{(\infty)}(\bar{G}), \partial^{k} v(0, y) / \partial x^{k}=0, y \in[0, b], k=$ $1,2, \ldots$

(2) $\left.v(x, y)\right|_{\Gamma}=0,\left.v(x, y)\right|_{\Gamma_{-\xi}}=\left.v(x, y)\right|_{\Gamma_{0}}$.

Theorem 3. The vector space $D_{A}(\bar{G})$ is dense in the space $H(\bar{G})$.

Thus, the operator $A$ acts from the dense vector space $D_{A}(\bar{G})$ of the Hilbert space $H(\bar{G})$ to the space $H(\bar{G})$.

Theorem 4. Operator $A$ is positively defined on the vector space $D_{A}(\bar{G})$.

To show the symmetry of the operator $A$ we use the following two lemmas, whose proofs are not difficult.

Lemma 5. For an arbitrary function $v(x, y)$ of the vector space $D_{A}(\bar{G})$ the following identity is valid:

$$
\mathscr{T} A v=A \mathscr{T} v .
$$


Lemma 6. For two arbitrary functions $v(x, y)$ and $w(x, y)$ of the vector space $D_{A}(\bar{G})$ we have

$$
\int_{-\xi}^{\xi} \frac{\partial \widetilde{v}(x, y)}{\partial x} \widetilde{w}(x, y) d x=0, \quad y \in[0, b] .
$$
form

The scalar product given by (5) can be represented in the

$$
\begin{aligned}
{[v, w]=} & \int_{0}^{b} \int_{-\xi}^{0} \int_{-a}^{x} v(s, y) w(s, y) d s d x d y \\
& +\xi \int_{0}^{b} \int_{-a}^{0} v(s, y) w(s, y) d x d y \\
& +\int_{0}^{b} \int_{0}^{\xi} \int_{-x}^{0}(2 v(0, y)-v(s, y)) \\
& \times(2 w(0, y)-w(s, y)) d s d x d y .
\end{aligned}
$$

In the case of the scalar product (5) we have the positively defined operator $A$, but it is not symmetric.

As $A$ is positive definite operator defined on the vector space $D_{A}(G)$ which is dense in the space $H(\bar{G})$, for the problem (1)-(3) we can use the standard way of the variational formulation [27].

Let us introduce the new scalar product on $D_{A}(\bar{G})$ :

$$
\begin{aligned}
{[v, w]_{A}=[A v, w] } & \\
=\int_{0}^{b} \int_{-\xi}^{\xi} \int_{-a}^{x}( & \frac{\partial \widetilde{v}(s, y)}{\partial s} \frac{\partial \widetilde{w}(s, y)}{\partial s} \\
& \left.+\frac{\partial \widetilde{v}(s, y)}{\partial y} \frac{\partial \widetilde{w}(s, y)}{\partial y}\right) d s d x d y .
\end{aligned}
$$

Denote by $\|\cdot\|_{A}$ the corresponding norm and by $\rho_{A}(v, w)$ the corresponding metric. By $H_{A}(\bar{G})$ we denote the space obtained after completion of $D_{A}(\bar{G})$ by the metric $\rho_{A}(v, w)$.

The following statement is true [24].

Theorem 7. The function $v(x, y) \in H(\bar{G})$ belongs to the space $H_{A}(\bar{G})$ if and only if the following relations are fulfilled:

$$
\begin{gathered}
v(x, y) \in H^{1}(G), \quad v(0, y) \in \stackrel{\circ}{H}^{1}(0, b), \\
\left.v(x, y)\right|_{\Gamma}=0,\left.\quad v(x, y)\right|_{\Gamma_{-\xi}}=\left.v(x, y)\right|_{\Gamma_{0}}=v(0, y) .
\end{gathered}
$$

Thus, functions of the space $H_{A}(\bar{G})$ satisfy boundary conditions. For every function $f(x, y) \in H(\bar{G})$ there exists a unique function $u(x, y)$ in the space $H_{A}(\bar{G})$, which minimizes the quadratic functional

$$
F(v)=\|v\|_{A}^{2}-2[f, v] .
$$

For any function $v(x, y) \in H_{A}(\bar{G})$ the following relation is fulfilled

$$
[u, v]_{A}=[f, v] .
$$

The function $u(x, y)$ from the space $H_{A}(\bar{G})$ which minimizes the functional (14) is called the generalized solution of the equation $A u=f$.

If the function $u(x, y)$ is sufficiently smooth then $u(x, y)$ is a solution in a classical sense of problem (1)-(3).

\section{Domain Decomposition and Sequential Algorithm}

In this section and next sections, for simplicity, let us consider Laplace equation with nonlocal (3) and again for simplicity homogeneous Dirichlet (2) conditions. So, we study the following problem:

$$
\begin{gathered}
\Delta u(x, y)=0, \quad(x, y) \in G, \\
\left.u(x, y)\right|_{\Gamma}=0, \\
\left.u(x, y)\right|_{\Gamma_{-\xi}}=\left.u(x, y)\right|_{\Gamma_{0}} .
\end{gathered}
$$

For problem (15)-(17) let us consider the following sequential iterative procedure:

$$
\begin{aligned}
& \Delta u_{1}^{n}(x, y)=0, \quad(x, y) \in G_{1}, \\
& \left.u_{1}^{n}(x, y)\right|_{\Gamma^{1}}=0, \\
& \left.u_{1}^{n}(x, y)\right|_{\Gamma_{-\xi_{1}}}=\left.u_{2}^{n-1}(x, y)\right|_{\Gamma_{-\xi_{1}}}, \\
& \quad n=1,2, \ldots ; \\
& \Delta u_{2}^{n}(x, y)=0, \quad(x, y) \in G_{2}, \\
& \left.u_{2}^{n}(x, y)\right|_{\Gamma^{2}}=0, \quad n=1,2, \ldots \\
& \left.u_{2}^{n}(x, y)\right|_{\Gamma_{-\xi}}=\left.u_{2}^{n}(x, y)\right|_{\Gamma_{0}}=\left.u_{1}^{n}(x, y)\right|_{\Gamma_{-\xi}}, \\
& n=1
\end{aligned}
$$

Here we utilize the following notations:

$$
\begin{gathered}
G_{1}=\left\{-a<x<-\xi_{1}, 0<y<b\right\}, \\
G_{2}=\{-\xi<x<0,0<y<b\},
\end{gathered}
$$

where $-\xi_{1}$ is a fixed point of the interval $(-\xi, 0), \Gamma^{1}=\partial G_{1} \backslash$ $\Gamma_{-\xi_{1}}, \Gamma^{2}=\partial G_{2} \backslash\left(\Gamma_{-\xi} \cup \Gamma_{0}\right)$, and $u_{2}^{0}\left(-\xi_{1}, y\right)=\varphi(y)$ is any continuous function on the segment $[0, b]$, which satisfies the following conditions: $\varphi(0)=\varphi(b)=0$.

The iterative procedure (18) reduces our nonlocal nonclassical problem (15)-(17) to the investigation of the sequence of classical Dirichlet boundary value problems on every step of the iteration.

The following statement takes place.

Theorem 8. The sequential iterative process (18) converges to a solution of problem (15)-(17) uniformly in the domain $\bar{G}$, and the following estimations are true:

$$
\begin{array}{ll}
\left|u(x, y)-u_{1}^{n}(x, y)\right| \leq C q^{n-1}, & (x, y) \in \bar{G}_{1}, \\
\left|u(x, y)-u_{2}^{n}(x, y)\right| \leq C q^{n-1}, & (x, y) \in \bar{G}_{2},
\end{array}
$$


where $q \in(0,1)$ is a constant independent of functions $u(x, y)$, $u_{1}^{n}(x, y), u_{2}^{n}(x, y)$, and constant $C$ depends on $\varphi(y)$.

Proof. Note that solving problem (18), we get two sequences $\left\{u_{1}^{n}\right\},\left\{u_{2}^{n}\right\}$ of harmonic functions, which are defined on the domains $\bar{G}_{1}$ and $\bar{G}_{2}$, respectively.

We have the following relations:

if $x=-\xi$ and $y \in[0, b]$, then $u_{2}^{n}=u_{1}^{n}$, that is $u_{1}^{n+1}-$ $u_{2}^{n}=u_{2}^{n+1}-u_{2}^{n}$

if $x=-\xi_{1}$ and $y \in[0, b]$, then $u_{2}^{n}=u_{1}^{n+1}$, that is $u_{1}^{n+1}-u_{1}^{n}=u_{2}^{n}-u_{2}^{n-1}$.

Let us introduce the notations:

$$
\begin{aligned}
M_{n} & =\max _{y \in[0, b]}\left|u_{1}^{n+1}(-\xi, y)-u_{1}^{n}(-\xi, y)\right| \\
& =\max _{y \in[0 ; b]}\left|u_{2}^{n+1}(-\xi, y)-u_{2}^{n}(-\xi, y)\right|, \\
M_{n}^{1} & =\max _{y \in[0 ; b]}\left|u_{1}^{n+1}\left(-\xi_{1}, y\right)-u_{1}^{n}\left(-\xi_{1}, y\right)\right| \\
& =\max _{y \in[0 ; b]}\left|u_{2}^{n}\left(-\xi_{1}, y\right)-u_{2}^{n-1}\left(-\xi_{1}, y\right)\right| .
\end{aligned}
$$

If $M_{n}^{1} \neq 0$, then, for the harmonic function $v_{1}^{n}=\left(u_{1}^{n+1}-\right.$ $\left.u_{1}^{n}\right) / M_{n}^{1}$, applying the lemma from [30, pages 250-254] to the domain $\bar{G}_{1}$, we have

$$
M_{n} \leq q M_{n}^{1}
$$

where $q \in(0,1)$ and depends only on domain $G_{1}$.

If $M_{n} \neq 0$, then, for the harmonic function $v_{2}^{n}=\left(u_{2}^{n+1}-\right.$ $\left.u_{2}^{n}\right) / M_{n}$, applying the extremum principle [30, pages 218 ] to the domain $G_{2}$, we have

$$
M_{n+1}^{1} \leq M_{n}
$$

If $M_{n}^{1}=0$ for some index $n$, then from the extremum principle we get $M_{n}=0$. So, the inequality (22) is also true.

If $M_{n}=0$ for some index $n$, then from the extremum principle we get again $M_{n+1}^{1}=0$. So, the inequality (23) is also true.

From the estimations (22) and (23) obtained above it follows that

$$
M_{n+1} \leq q M_{n}, \quad M_{n+1}^{1} \leq q M_{n}^{1} .
$$

This means that the sequences $\left\{M_{n}\right\}$ and $\left\{M_{n}^{1}\right\}$ tend to zero, and we obtain the uniform convergence of the series

$$
\begin{aligned}
& u_{1}^{1}+\sum_{k=1}^{\infty}\left(u_{1}^{k+1}-u_{1}^{k}\right)=\lim _{n \rightarrow \infty} u_{1}^{n}=u_{1}, \\
& u_{2}^{1}+\sum_{k=1}^{\infty}\left(u_{2}^{k+1}-u_{2}^{k}\right)=\lim _{n \rightarrow \infty} u_{2}^{n}=u_{2},
\end{aligned}
$$

in the domains $\bar{G}_{1}$ and $\bar{G}_{2}$, respectively.
According to Weierstrass theorem [30, pages 232, 233], the functions $u_{1}$ and $u_{2}$ are harmonic ones, defined, respectively, in $G_{1}$ and $G_{2}$, and satisfy the condition (16). As for domain $G_{12}=G_{1} \cap G_{2}$, we have the following relations:

$$
\begin{aligned}
& \text { if } x=-\xi \text { and } y \in[0, b] \text {, then } u_{2}^{n}-u_{1}^{n}=0 ; \\
& \text { if } x=-\xi_{1} \text { and } y \in[0, b] \text {, then } u_{2}^{n}-u_{1}^{n}=u_{2}^{n}-u_{2}^{n-1} \text {. }
\end{aligned}
$$

The latter difference tends to zero uniformly.

Again, according to the extremum principle, we obtain that the functions $u_{1}$ and $u_{2}$ coincide with each other in the domain $G_{12}$ and define a regular harmonic function on $G$, which represents the solution of the problem (15)-(17).

Now, let us estimate the rate of convergence of the iterative process (18). Using the triangle inequality, the extremum principle and the second inequality of (24), we have

$$
\begin{aligned}
\left|u_{1}^{n+p}-u_{1}^{n}\right|= & \mid u_{1}^{n+p}-u_{1}^{n+p-1}+u_{1}^{n+p-1}-u_{1}^{n+p-2} \\
& +\cdots+u_{1}^{n+2}-u_{1}^{n+1}+u_{1}^{n+1}-u_{1}^{n} \mid \\
\leq & M_{n+p-1}^{1}+M_{n+p-2}^{1}+\cdots+M_{n+1}^{1}+M_{n}^{1} \\
\leq & q^{n+p-2} M_{1}^{1}+q^{n+p-3} M_{1}^{1}+\cdots+q^{n} M_{1}^{1}+q^{n-1} M_{1}^{1} \\
= & q^{n-1} M_{1}^{1}\left(q^{p-1}+q^{p-2}+\cdots+q+1\right) \\
= & q^{n-1} M_{1}^{1} \frac{1-q^{p}}{1-q}, \\
M_{1}^{1}= & \max _{y \in[0 ; b]}\left|u_{1}^{2}\left(-\xi_{1}, y\right)-u_{1}^{1}\left(-\xi_{1}, y\right)\right| \\
\leq & \max _{y \in[0 ; b]}\left(\left|u_{1}^{2}\left(-\xi_{1}, y\right)\right|+\left|u_{1}^{1}\left(-\xi_{1}, y\right)\right|\right) \\
= & \max _{y \in[0 ; b]}\left|u_{2}^{1}\left(-\xi_{1}, y\right)\right|+\max _{y \in[0 ; b]}\left|u_{2}^{0}\left(-\xi_{1}, y\right)\right| \\
\leq & 2 \max _{y \in[0 ; b]}|\varphi(y)|=M .
\end{aligned}
$$

Thus,

$$
\left|u_{1}^{n+p}-u_{1}^{n}\right| \leq q^{n-1} M \frac{1-q^{p}}{1-q} .
$$

If in this inequality we tend $p$ to $\infty$, then we obtain in the domain $\bar{G}_{1}$

$$
\left|u_{1}-u_{1}^{n}\right| \leq q^{n-1} \frac{M}{1-q}
$$

Analogous estimation is true for $u_{2}$ in the domain $\bar{G}_{2}$. Consequently, we get

$$
\begin{aligned}
& \left|u_{1}(x, y)-u_{1}^{n}(x, y)\right| \leq C q^{n-1}, \quad(x, y) \in \bar{G}_{1}, \\
& \left|u_{2}(x, y)-u_{2}^{n}(x, y)\right| \leq C q^{n-1}, \quad(x, y) \in \bar{G}_{2} .
\end{aligned}
$$

This completes the proof of Theorem 8 . 


\section{Domain Decomposition and Parallel Algorithm}

Algorithm (18) for the solution of the problem (15) has a sequential form. Now, let us consider one more approach to the solution of the problem (15)-(17). In this case the search of approximate solutions on domains $\bar{G}_{1}$ and $\bar{G}_{2}$ will be carried out not by means of a sequential algorithm but in a parallel way. cess:

Consider the following overlapping parallel iterative pro-

$$
\begin{aligned}
& \Delta u_{1}^{n}(x, y)=0, \quad(x, y) \in G_{1}, \\
& \left.u_{1}^{n}(x, y)\right|_{\Gamma^{1}}=0, \\
& \left.u_{1}^{n}(x, y)\right|_{\Gamma_{-\xi_{1}}}=\left.u_{2}^{n-1}(x, y)\right|_{\Gamma_{-\xi_{1}}}, \\
& n=1,2, \ldots ; \\
& \Delta u_{2}^{n}(x, y)=0, \quad(x, y) \in G_{2}, \\
& \left.u_{2}^{n}(x, y)\right|_{\Gamma^{2}}=0, \quad n=1,2, \ldots, \\
& \left.u_{2}^{n}(x, y)\right|_{\Gamma_{-\xi}}=\left.u_{2}^{n}(x, y)\right|_{\Gamma^{0}}=\left.u_{1}^{n-1}(x, y)\right|_{\Gamma_{-\xi}},
\end{aligned}
$$

where $u_{2}^{0}\left(-\xi_{1}, y\right)=\varphi_{1}(y)$ and $u_{1}^{0}(\xi, y)=\varphi_{2}(y)$ are any continuous functions on the segment $[0, b]$, which satisfy the following conditions: $\varphi_{1}(0)=\varphi_{1}(b)=0, \varphi_{2}(0)=\varphi_{2}(b)=0$.

The following statement takes place.

Theorem 9. The parallel iterative process (30) converges to a solution of the problem (15)-(17) uniformly in the domain $\bar{G}$, and the following estimations are true:

$$
\begin{array}{ll}
\left|u(x, y)-u_{1}^{n}(x, y)\right| \leq C q^{(n / 2)-1}, & (x, y) \in \bar{G}_{1}, \\
\left|u(x, y)-u_{2}^{n}(x, y)\right| \leq C q^{(n / 2)-1}, & (x, y) \in \bar{G}_{2},
\end{array}
$$

where $q \in(0,1)$ is a constant independent of functions $u(x, y)$, $u_{1}^{n}(x, y)$, and $u_{2}^{n}(x, y)$, and constant $C$ depends on $\varphi_{1}(y)$, $\varphi_{2}(y)$.

Proof. Let us prove this theorem in a similar way as Theorem 8 was proven. We should note that the boundary value problems (30) are simultaneously solved in domains $G_{1}$ and $G_{2}$, respectively. Sequences of harmonic functions $\left\{u_{1}^{n}\right\}$, $\left\{u_{2}^{n}\right\}$ are defined on $\bar{G}_{1}$ and $\bar{G}_{2}$, respectively.

The following relations are satisfied:

$$
\begin{aligned}
& \text { if } x=\xi \text { and } y \in[0 ; b] \text {, then } u_{2}^{n}=u_{1}^{n-1} \text {; that is, } u_{1}^{n}- \\
& u_{1}^{n-1}=u_{2}^{n+1}-\bar{u}_{2}^{n} \text {; } \\
& \text { if } x=-\xi_{1} \text { and } y \in[0 ; b] \text {, then } u_{2}^{n-1}=u_{1}^{n} \text {; that is, } \\
& u_{1}^{n+1}-u_{1}^{n}=u_{2}^{n}-u_{2}^{n-1} \text {. }
\end{aligned}
$$

If we introduce the notation

$$
\begin{aligned}
M_{n-1} & =\max _{y \in[0 ; b]}\left|u_{1}^{n}(-\xi, y)-u_{1}^{n-1}(-\xi, y)\right| \\
& =\max _{y \in[0 ; b]}\left|u_{2}^{n+1}(-\xi, y)-u_{2}^{n}(-\xi, y)\right|, \\
M_{n}^{1} & =\max _{y \in[0 ; b]}\left|u_{1}^{n+1}\left(-\xi_{1}, y\right)-u_{1}^{n}\left(-\xi_{1}, y\right)\right| \\
& =\max _{y \in[0 ; b]}\left|u_{2}^{n}\left(-\xi_{1}, y\right)-u_{2}^{n-1}\left(-\xi_{1}, y\right)\right|,
\end{aligned}
$$

and, for the harmonic function $v_{1}^{n}=\left(u_{1}^{n+1}-u_{1}^{n}\right) / M_{n}^{1}$, apply the lemma from [30, pages 250-254] to the domain $G_{1}$, we will have the estimation

$$
M_{n} \leq q M_{n}^{1},
$$

where $q \in(0,1)$ and depends on domain $G_{1}$ only.

If $M_{n}^{1}=0$, then from the extremum principle we get that $M_{n}=0$ and the inequality (33) is clear.

If $M_{n} \neq 0$, then, for the harmonic function $v_{2}^{n-1}=\left(u_{2}^{n+1}-\right.$ $\left.u_{2}^{n}\right) / M_{n-1}$, from the extremum principle in the domain $G_{2}$ we have

$$
M_{n+1}^{1} \leq M_{n-1} .
$$

If $M_{n-1}=0$, then, using again the extremum principle, we have $M_{n}^{1}=0$ and the inequality (34) holds.

From the estimations (33) and (34), we obtain

$$
M_{n+1} \leq q M_{n-1}, \quad M_{n+2}^{1} \leq q M_{n}^{1} .
$$

That means that the sequences $\left\{M_{n}\right\}$ and $\left\{M_{n}^{1}\right\}$ tend to zero.

Thus, in this case, the series analogous to the series from (25) are also uniformly convergent. The corresponding harmonic functions $u_{1}$ and $u_{2}$ are defined in $\bar{G}_{1}$ and $\bar{G}_{2}$, respectively, and satisfy condition (16). As for the common part of these domains, we have

$$
\begin{aligned}
& \text { if } x=-\xi \text { and } y \in[0, b] \text {, then } u_{2}^{n}-u_{1}^{n}=u_{2}^{n}-u_{2}^{n+1} \text {; } \\
& \text { if } x=-\xi_{1} \text { and } y \in[0, b] \text {, then } u_{2}^{n}-u_{1}^{n}=u_{2}^{n}-u_{2}^{n-1}
\end{aligned}
$$

and this difference tends to zero, when $n \rightarrow \infty$. Thus, the functions $u_{1}$ and $u_{2}$ again coincide in the domain $G_{12}$ and define a regular harmonic function on $G$, which represents the solution of the problem (15)-(17).

Let us estimate the rate of convergence of the constructed sequences.

We should remark that from the second inequality of (35) we have the following: if $n=2 k$, then

$$
M_{n}^{1} \leq q^{(n-2) / 2} M_{2}^{1}
$$

if $n=2 k-1$, then

$$
M_{n}^{1} \leq q^{(n-1) / 2} M_{1}^{1} .
$$

Using the triangle inequality, we get

$$
\begin{aligned}
\left|u_{1}^{n+p}-u_{1}^{n}\right|= & \mid u_{1}^{n+p}-u_{1}^{n+p-1}+u_{1}^{n+p-1}-u_{1}^{n+p-2} \\
& +\cdots+u_{1}^{n+2}-u_{1}^{n+1}+u_{1}^{n+1}-u_{1}^{n} \mid \\
\leq & M_{n+p-1}^{1}+M_{n+p-2}^{1}+\cdots+M_{n+1}^{1}+M_{n}^{1} .
\end{aligned}
$$


Taking into account inequalities (36) and (37) for $n=2 k$ and $p=2 m$, we obtain from (36)

$$
\begin{aligned}
\mid u_{1}^{n+p} & -u_{1}^{n} \mid \\
\leq & q^{(n+p-1-1) / 2} M_{1}^{1}+q^{(n+p-2-2) / 2} M_{2}^{1} \\
& +q^{(n+p-3-1) / 2} M_{1}^{1}+q^{(n+p-4-2) / 2} M_{2}^{1} \\
& +\cdots+q^{(n+1-1) / 2} M_{1}^{1}+q^{(n-2) / 2} M_{2}^{1} \\
= & \left(M_{1}^{1}+M_{2}^{1}\right) q^{n / 2}\left(q^{(p-4) / 2}+q^{(p-6) / 2}+\cdots+q+1\right) \\
& +q^{(n+p-2) / 2} M_{1}^{1}+q^{(n-2) / 2} M_{2}^{1} \\
= & q^{(n-2) / 2}\left[q\left(M_{1}^{1}+M_{2}^{1}\right) \frac{1-q^{(p-2) / 2}}{1-q}+q^{p / 2} M_{1}^{1}+M_{2}^{1}\right] .
\end{aligned}
$$

If $n=2 k-1$ and $p=2 m-1$, then from (38) we have

$$
\begin{aligned}
\left|u_{1}^{n+p}-u_{1}^{n}\right| \leq & q^{(n+p-1-1) / 2} M_{1}^{1}+q^{(n+p-2-2) / 2} M_{2}^{1} \\
& +q^{(n+p-3-1) / 2} M_{1}^{1}+q^{(n+p-4-2) / 2} M_{2}^{1} \\
& +\cdots+q^{(n+1-2) / 2} M_{2}^{1}+q^{(n-1) / 2} M_{1}^{1} \\
= & q^{(n+p-1) / 2} M_{1}^{1}+\left(M_{1}^{1}+M_{2}^{1}\right) q^{(n-2) / 2} \\
& \times\left(q^{(p-2) / 2}+q^{(p-4) / 2}+\cdots+q^{3 / 2}+q^{1 / 2}\right) \\
= & q^{(n-2) / 2}\left[q^{p / 2} M_{1}^{1}+\left(M_{1}^{1}+M_{2}^{1}\right) \frac{q^{1 / 2}-q^{p / 2}}{1-q}\right] .
\end{aligned}
$$

$$
\text { If } n=2 k \text { and } p=2 m-1 \text {, then }
$$

$$
\begin{aligned}
\left|u_{1}^{n+p}-u_{1}^{n}\right| \leq & q^{(n+p-1-2) / 2} M_{2}^{1}+q^{(n+p-2-1) / 2} M_{1}^{1} \\
& +\cdots+q^{(n+3-1) / 2} M_{1}^{1}+q^{(n+2-2) / 2} M_{2}^{1} \\
& +q^{(n+1-1) / 2} M_{1}^{1}+q^{(n-2) / 2} M_{2}^{1} \\
= & q^{(n-2) / 2}\left[( M _ { 1 } ^ { 1 } + M _ { 2 } ^ { 1 } ) \left(q^{(p-1) / 2}+q^{(p-3) / 2}\right.\right. \\
& \left.+\cdots+q)+M_{2}^{1}\right] \\
= & q^{(n-2) / 2}\left[\left(M_{1}^{1}+M_{2}^{1}\right) \frac{q-q^{(p+1) / 2}}{1-q}+M_{2}^{1}\right],
\end{aligned}
$$

and at last, supposing $n=2 k-1$ and $p=2 m$, we have

$$
\begin{aligned}
\left|u_{1}^{n+p}-u_{1}^{n}\right| \leq & q^{(n+p-1-2) / 2} M_{2}^{1}+q^{(n+p-2-1) / 2} M_{1}^{1} \\
& +\cdots+q^{(n+3-2) / 2} M_{2}^{1}+q^{(n+2-1) / 2} M_{1}^{1} \\
& +q^{(n+1-2) / 2} M_{2}^{1}+q^{(n-1) / 2} M_{1}^{1} \\
= & q^{(n-2) / 2}\left(M_{1}^{1}+M_{2}^{1}\right) q^{(n-2) / 2} \\
& \times\left(q^{(p-1) / 2}+q^{(p-3) / 2}+\cdots+q^{3 / 2}+q^{1 / 2}\right) \\
= & q^{(n-2) / 2}\left(M_{1}^{1}+M_{2}^{1}\right) \frac{q^{1 / 2}-q^{(p+1) / 2}}{1-q} .
\end{aligned}
$$

If in the obtained inequalities we tend to the limit, when $p \rightarrow \infty$, we get the following estimations.

If $n=2 k$ and $p=2 m$, then

$$
\left|u-u_{1}^{n}\right| \leq q^{(n-2) / 2}\left[\frac{\left(M_{1}^{1}+M_{2}^{1}\right) q}{1-q}+M_{2}^{1}\right] ;
$$

if $n=2 k-1$ and $p=2 m-1$, then

$$
\left|u-u_{1}^{n}\right| \leq q^{(n-1) / 2} \frac{\left(M_{1}^{1}+M_{2}^{1}\right)}{1-q} ;
$$

if $n=2 k$ and $p=2 m-1$, then

$$
\left|u-u_{1}^{n}\right| \leq q^{(n-2) / 2}\left[\frac{\left(M_{1}^{1}+M_{2}^{1}\right) q}{1-q}+M_{2}^{1}\right] ;
$$

and if $n=2 k-1$ and $p=2 m$, then

$$
\left|u-u_{1}^{n}\right| \leq q^{(n-1) / 2} \frac{\left(M_{1}^{1}+M_{2}^{1}\right)}{1-q} .
$$

Let us estimate $M_{1}^{1}$ and $M_{2}^{1}$. We have

$$
\begin{aligned}
M_{1}^{1} & =\max _{y \in[0 ; b]}\left|u_{1}^{2}\left(-\xi_{1}, y\right)-u_{1}^{1}\left(-\xi_{1}, y\right)\right| \\
& \leq \max _{y \in[0 ; b]}\left(\left|u_{1}^{2}\left(-\xi_{1}, y\right)\right|+\left|u_{1}^{1}\left(-\xi_{1}, y\right)\right|\right) \\
& =\max _{y \in[0 ; b]}\left(\left|u_{2}^{1}\left(-\xi_{1}, y\right)\right|+\left|u_{2}^{0}\left(-\xi_{1}, y\right)\right|\right) \\
& \leq 2 \max _{y \in[0 ; b]}\left\{\left|\varphi_{1}(y)\right|,\left|\varphi_{2}(y)\right|\right\}, \\
M_{2}^{1} & =\max _{y \in[0 ; b]}\left|u_{1}^{3}\left(-\xi_{1}, y\right)-u_{1}^{2}\left(-\xi_{1}, y\right)\right| \\
& \leq \max _{y \in[0 ; b]}\left(\left|u_{1}^{3}\left(-\xi_{1}, y\right)\right|+\left|u_{1}^{2}\left(-\xi_{1}, y\right)\right|\right) \\
& =\max _{y \in[0 ; b]}\left(\left|u_{1}^{2}\left(-\xi_{1}, y\right)\right|+\left|u_{1}^{1}\left(-\xi_{1}, y\right)\right|\right) \\
& \leq 2 \max _{y \in[0 ; b]}\left\{\left|\varphi_{1}(y)\right|,\left|\varphi_{2}(y)\right|\right\} .
\end{aligned}
$$


So, for any $n$, we obtain the following relation in the domain $\bar{G}_{1}$ :

$$
\left|u_{1}(x, y)-u_{1}^{n}(x, y)\right| \leq C q^{(n-2) / 2} .
$$

Analogous estimation is true for $u_{2}$ in the domain $\bar{G}_{2}$

$$
\left|u_{2}(x, y)-u_{2}^{n}(x, y)\right| \leq C q^{(n-2) / 2} .
$$

Theorems similar to Theorems 8 and 9 are valid for the sequential as well as parallel algorithms for multigrid domain decomposition case too.

\section{Conclusion}

Because of importance of nonlocal problems many scientific articles are devoted for their investigation. We gave one problem to illustrate the variational formulation and domain decomposition of the problem. Nonlocal problems for the second and the fourth order ordinary and partial differential equations are also studied by authors. Nonlocal boundary value problems with some kind of nonlocal integral conditions are studied as well (see, e.g., [15, 24-26] and references therein).

\section{Conflict of Interests}

The authors declare that there is no conflict of interests regarding the publication of this paper.

\section{Acknowledgments}

The first author thanks Fulbright Visiting Scholar Program (Grant no. AY 2012-2013, USA) and the Shota Rustaveli National Science Foundation (Grant no. DI/16/4-120/11, Georgia) for the financial support and the Naval Postgraduate School in Monterey, CA, USA, for hosting him during the nine months of his tenure in 2012-2013. The second author thanks the Shota Rustaveli National Scientific Foundation (Grant no. YS/40/5-106/12, Georgia) for the financial support and the Naval Postgraduate School in Monterey, CA, USA, for hosting him during the four months of his tenure in 2013.

\section{References}

[1] A. V. Bitsadze and A. A. Samarskii, "On some simplified generalization of the linear elliptic problems," Doklady Akademii Nauk SSSR, vol. 185, pp. 739-740, 1969 (Russian).

[2] A. Ashyralyev, "On well-posedness of the nonlocal boundary value problems for elliptic equations," Numerical Functional Analysis and Optimization, vol. 24, no. 1-2, pp. 1-15, 2003.

[3] A. Ashyralyev and E. Ozturk, "The numerical solution of the Bitsadze-Samarskii nonlocal boundary value problems with the Dirichlet-Neumann condition," Abstract and Applied Analysis, vol. 2012, Article ID 730804, 13 pages, 2012.

[4] A. Ashyralyev, O. Tetikoglu, and F. Songul, "FDM for elliptic equations with Bitsadze-Samarskii-Dirichlet conditions," Abstract and Applied Analysis, vol. 2012, Article ID 454831, 22 pages, 2012.
[5] G. Berikelashvili, D. Gordeziani, and S. Kharibegashvili, "Finite difference scheme for one mixed problem with integral condition," in Proceedings of the 2nd WSEAS International Conference on Finite Differences, Finite Elements, Finite Volumes, Boundary Elements, pp. 118-120, 2009.

[6] D. G. Gordeziani, "On one method of solving the BitsadzeSamarskii boundary value problem," in Proceedings of the Industrial and Applied Mathematics Seminar, pp. 39-41, 1970, 1970 (Russian).

[7] T. A. Jangveladze, "On one iterative method of solution of Bitsadze-Samarskii boundary value problem," Manuscript, Faculty of Applied Mathematics and Cybernetics of Tbilisi State University, Tbilisi, Georgia, 1975 (Georgian).

[8] D. G. Gordeziani, On the Methods of Solution for One Class of Non-Local Boundary Value Problems, University Press, Tbilisi, Georgia, 1981 (Russian).

[9] A. K. Gushin and V. P. Mikhailov, "On the continuity of the solutions of a class of non-local problems for an elliptic equation," Sbornik: Mathematics, vol. 186, no. 2, pp. 197-219, 1995 (Russian).

[10] V. A. Il'in and E. I. Moiseev, “Two-dimensional nonlocal boundary value problem for Poisson's operator in the differential and difference forms," Mathematical Modelling, vol. 2, pp. 139-156, 1990 (Russian).

[11] T. A. Jangveladze, "Domain decomposition method for Bitsadze-Samarskii boundary value problem for second order nonlinear elliptic equation," in Proceedings of the Postgraduate Students' Scientific Conference of Ivane Javakhishvili Tbilisi State University, p. 7, Tbilisi State University, Tbilisi, Georgia, 1977.

[12] T. A. Jangveladze and Z. V. Kiguradze, "Domain decomposition for Bitsadze-Samarskii boundary value problem," Reports of Enlarged Sessions of the Seminar of I. Vekua Institute of Applied Mathematics, vol. 16, pp. 16-19, 2001.

[13] T. Jangveladze, Z. Kiguradze, and G. Lobjanidze, "On decomposition methods and variational formulation for Bitsadze-Samarskii nonlocal boundary value problem for twodimensional second order elliptic equations," in Proccedings of the 15th WSEAS International Conference on Applied Mathematics (MATH '10), pp. 116-121, Athens, Greece, December 2010.

[14] J. Mo and C. Ouyang, "A class of nonlocal boundary value problems of nonlinear elliptic systems in unbounded domains," Acta Mathematica Scientia, vol. 21, no. 1, pp. 93-97, 2001.

[15] Z. V. Kiguradze, "Domain decomposition and parallel algorithm for Bitsadze-Samarskii boundary value problem," Reports of Enlarged Sessions of the Seminar of I. Vekua Institute of Applied Mathematics, vol. 10, pp. 49-51, 1995.

[16] B. P. Paneyakh, "On some nonlocal boundary value problems for linear differential operators," Matematicheskie Zametki, vol. 35, no. 3, pp. 425-433, 1984 (Russian).

[17] M. P. Sapagovas and P. U. Chegis, "On some boundary value problems with nonlocal conditions," Differential Equations, vol. 23, pp. 1268-1274, 1987 (Russian).

[18] A. L. Skubachevskii, "On a spectrum of some nonlocal elliptic boundary value problems," Sbornik: Mathematics, vol. 117, pp. 548-558, 1982 (Russian).

[19] T. A. Jangveladze and G. B. Lobjanidze, "On a variational statement of a nonlocal boundary value problem for a fourth-order ordinary differential equation," Differentsial'nye Uravneniya, vol. 45, no. 3, pp. 325-333, 2009 (Russian), English translation in Differential Equations vol. 45, no. 3, pp. 335-343, 2009. 
[20] T. A. Jangveladze and G. B. Lobjanidze, "On a nonlocal boundary value problem for a fourth-order ordinary differential equation," Differentsial'nye Uravneniya, vol. 47, no. 2, pp. 181188, 2011 (Russian), English translation in Differential Equations vol. 47, no. 2, pp. 179-186, 2011.

[21] G. L. Karakostas and P. Ch. Tsamatos, "Sufficient conditions for the existence of nonnegative solutions of a nonlocal boundary value problem," Applied Mathematics Letters, vol. 15, no. 4, pp. 401-407, 2002.

[22] I. Kiguradze and T. Kiguradze, "Conditions for the wellposedness of nonlocal problems for higher order linear differential equations with singularities," Georgian Mathematical Journal, vol. 18, no. 4, pp. 735-760, 2011.

[23] P. L. Lions, "On the Schwarz alternating method I," in First International Symposium on Domain Decomposition Methods for Partial Differential Equations, R. Glowinski, G. H. Golub, G. A. Meurant, and J. Periaux, Eds., pp. 2-42, SIAM, Philadelphia, Pa, USA, 1988.

[24] G. Lobjanidze, "On the variational formulation of BitsadzeSamarskii problem for the equation $-\Delta u+\lambda u=f$," Reports of Enlarged Sessions of the Seminar of I. Vekua Institute of Applied Mathematics, vol. 18, pp. 39-42, 2003.

[25] G. Lobjanidze, "On variational formulation of some nonlocal boundary value problems by symmetric continuation operation of a function," Journal of Applied Mathematics and Mechanics, vol. 12, pp. 15-22, 2006.

[26] G. Lobjanidze, "On variational formulation of some nonlocal boundary value problem," Reports of Enlarged Sessions of the Seminar of I. Vekua Institute of Applied Mathematics, vol. 25, pp. 80-85, 2011.

[27] K. Rektorys, Variational Methods in Mathematics, Science and Engineering, Springer, Prague, Czech Republic, 1980.

[28] S. Nepomnyaschikh, "Domain decomposition methods," Radon Series on Computational and Applied Mathematics, vol. 1, pp. 89-160, 2007.

[29] A. Quarteroni and A. Valli, Domain Decomposition Methods for Partial Differential Equations, Oxford University Press, Oxford, UK, 1999.

[30] R. Courant and D. Hilbert, Methods of Mathematical Physics, vol. 2, Leningrad, Moscow, Russia, 1951 (Russian).

[31] Ch. P. Gupta and S. I. Trofimchuk, "A sharper condition for the solvability of a three-point second order boundary value problem," Journal of Mathematical Analysis and Applications, vol. 205, no. 2, pp. 586-597, 1997.

[32] A. Lomtatidze and L. Malaguti, "On a nonlocal boundary value problem for second order nonlinear singular differential equations," Georgian Mathematical Journal, vol. 7, no. 1, pp. 133154, 2000.

[33] R. Ma and N. Castaneda, "Existence of solutions of nonlinear m-point boundary-value problems," Journal of Mathematical Analysis and Applications, vol. 256, no. 2, pp. 556-567, 2001. 


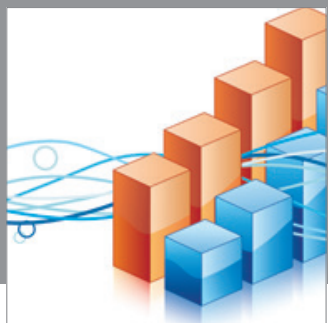

Advances in

Operations Research

mansans

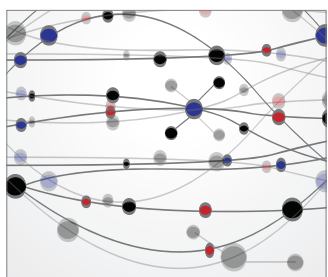

The Scientific World Journal
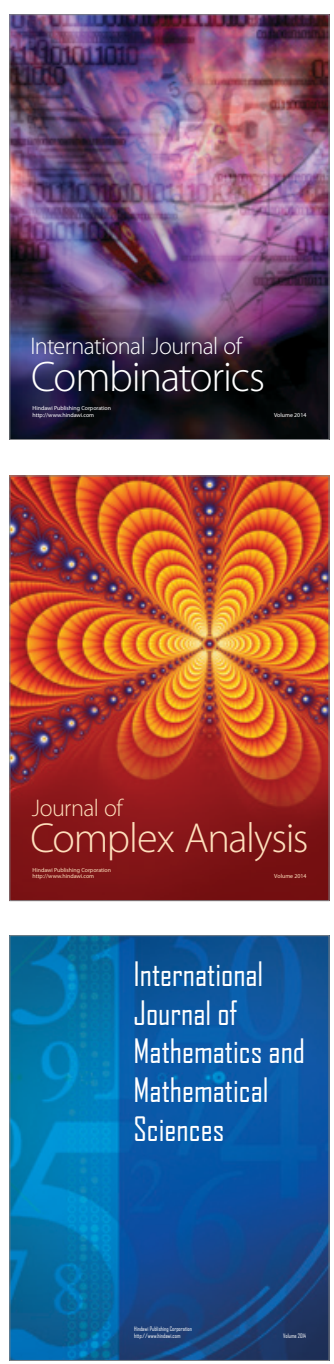
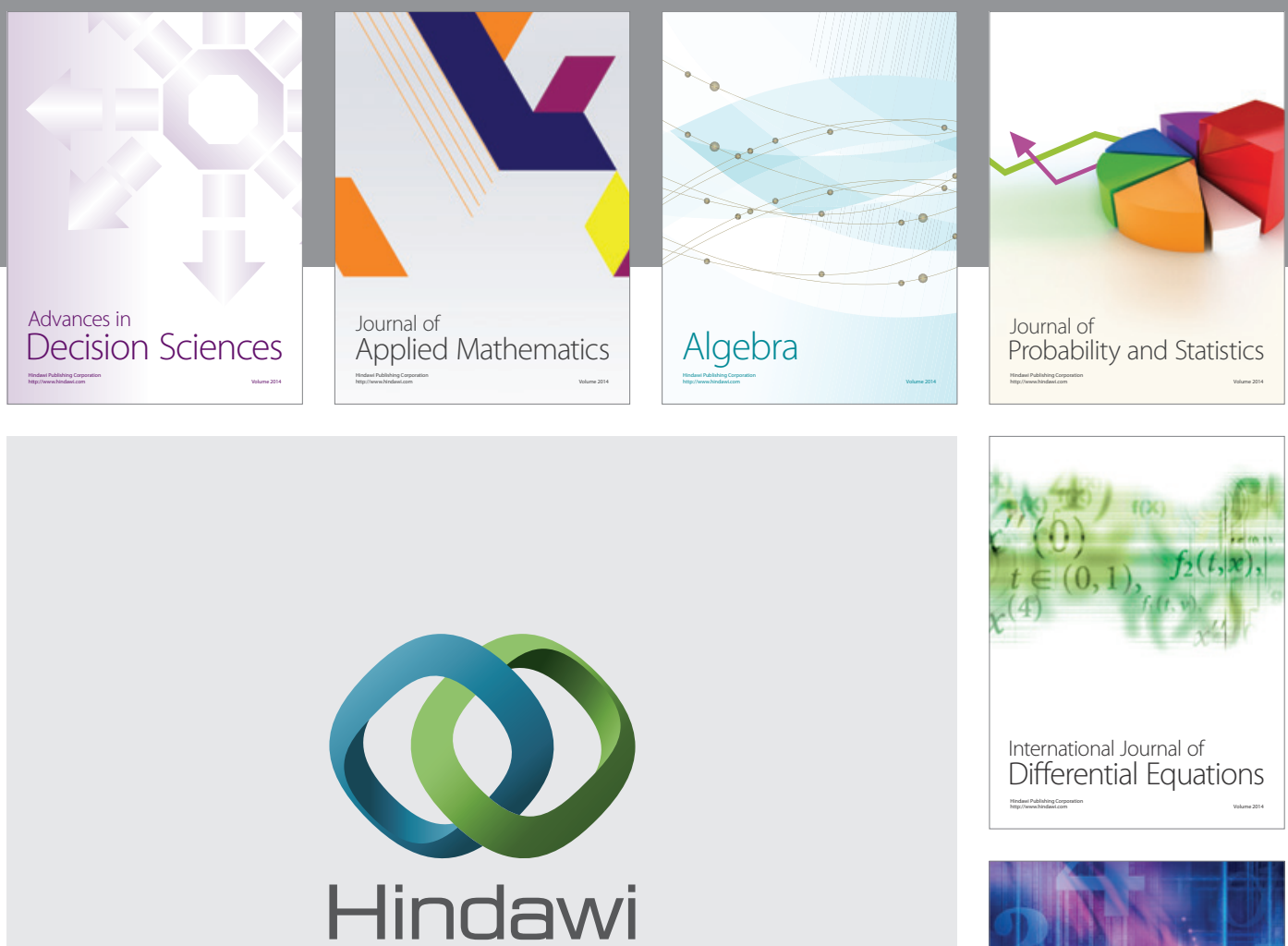

Submit your manuscripts at http://www.hindawi.com
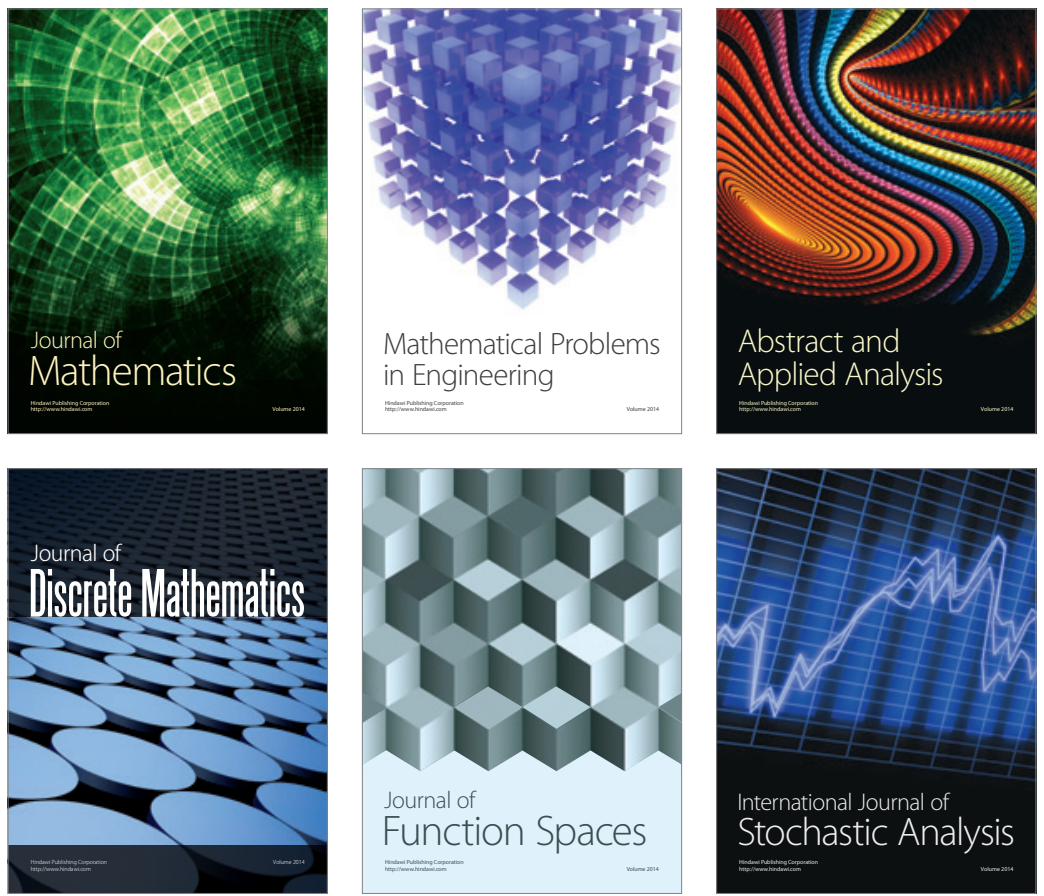

Journal of

Function Spaces

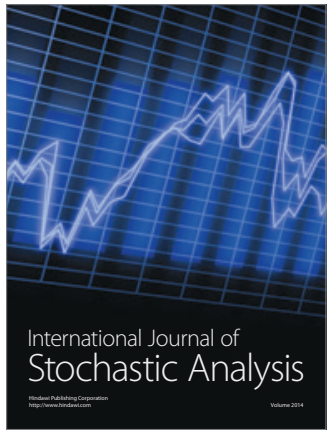

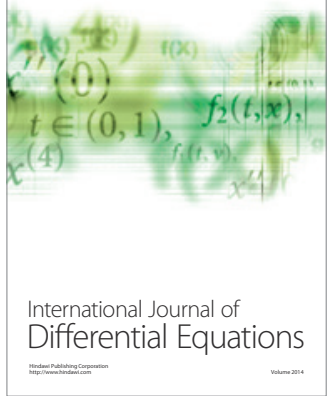
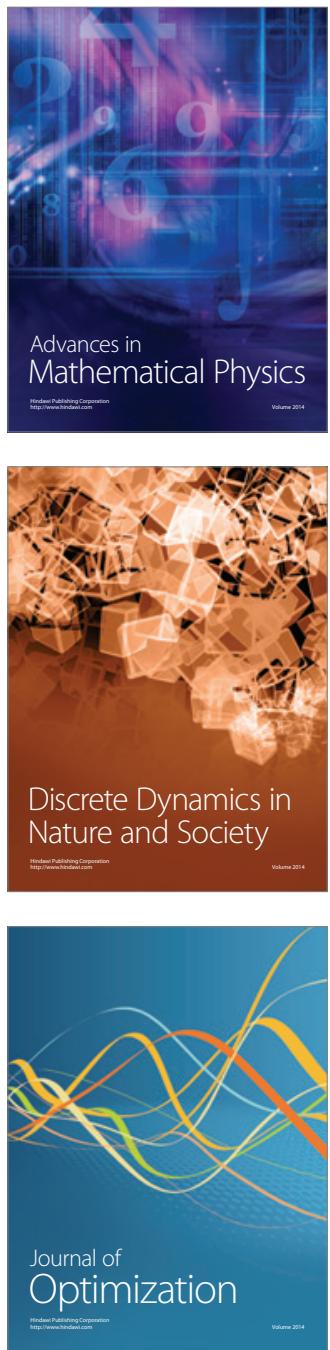